\title{
Efficient Low Dissipative High Order Schemes for Multiscale MHD Flows, I: Basic Theory
}

\author{
Björn Sjögreen ${ }^{1}$ and H. C. Yee ${ }^{2}$ \\ 1 Royal Institute of Technology, Sweden bjorns@nada.kth.se \\ 2 NASA Ames Research Center yee@nas.nasa.gov
}

\section{Abstract}

The objective of this paper is to extend our recently developed highly parallelizable nonlinear stable high order schemes for complex multiscale hydrodynamic applications to the viscous MHD equations. These schemes employed multiresolution wavelets as adaptive numerical dissipation controls to limit the amount of and to aid the selection and/or blending of the appropriate types of dissipation to be used. The new scheme is formulated for both the conservative and non-conservative form of the MHD equations in curvilinear grids. The four advantages of the present approach over existing MHD schemes reported in the open literature are as follows. First, the scheme is constructed for long-time integrations of shock/turbulence/combustion MHD flows. Available schemes are too diffusive for long-time integrations and/or turbulence/combustion problems. Second, unlike existing schemes for the conservative MHD equations which suffer from ill-conditioned eigendecompositions, the present scheme makes use of a well-conditioned eigen-decomposition obtained from a minor modification of the eigenvectors of the non-conservative MHD equations to solve the conservative form of the MHD equations. Third, this approach of using the non-conservative eigensystem when solving the conservative equations also works well in the context of standard shock-capturing schemes for the MHD equations. Fourth, a new approach to minimize the numerical error of the divergence-free magnetic condition for high order schemes is introduced. Numerical experiments with typical MHD model problems revealed the applicability of the newly developed schemes for the MHD equations.

\section{Motivation \& Relevance}

Accurate numerical simulations of complex multiscale compressible viscous flows, especially high speed turbulence combustion and acoustics, demand high order schemes with adaptive numerical dissipation controls. Standard high resolution shock-capturing methods are too dissipative to capture the small scales and/or long-time wave propagations without extreme grid refinements and small time steps. An integrated approach for the control of numerical dissipation in high order schemes for the compressible Euler and Navier-Stokes equations has been developed and verified by the authors and collaborators $[18,19,12,20,15]$. These schemes are suitable for the problems in question. Basically, the scheme consists of sixth-order or higher non-dissipative spatial difference operators as the base scheme. To control the amount and types of numerical dissipation, an artificial compression method (ACM) sensor or multiresolution wavelets are used as sensors to adaptively limit the amount and to aid in the selection and/or blending of the appropriate types of numerical dissipation to be used. This adaptive control of numerical dissipation 
is accomplished by a filter step after the completion of each full time step integration of the base scheme.

MHD and/or plasma injections into a gas dynamics field could play an important role in drag reduction in highly maneuverable high speed combat aircraft. MHD plays a key role in space weather forecasting, and in the understanding of the dynamics of the evolution of our solar system and the main sequence stars, especially certain types of star formation. Although there exist a few well-studied second- and third-order high-resolution shockcapturing schemes for MHD in the open literature, these schemes are too diffusive and not practical for turbulence/combustion MHD flows. On the other hand, extension of higher than third-order high-resolution schemes to the MHD system of equations is not straightforward. Unlike the hydrodynamic equations, the inviscid MHD system is non-strictly hyperbolic with non-convex fluxes. The wave structures and shock types are different from their hydrodynamic counterparts. Many of the non-traditional hydrodynamic shocks are not fully understood. Consequently, reliable and highly accurate numerical schemes for multiscale MHD equations pose a great challenge to algorithm development. In addition, controlling the numerical error of the divergence-free condition of the magnetic field for high order methods has been a stumbling block. Lower order methods are not practical for the astrophysical problems in question. We propose to extend our hydrodynamics schemes to the MHD equations, gaining several desirable advantages over commonly used MHD schemes.

The present paper is Part I of a series of papers on the subject. Here, we present our new scheme with typical ideal MHD test cases to validate our approach and to illustrate the representative performance of the scheme. Since there are many variants in the minimization of the divergence of the magnetic field $(\nabla \cdot \mathbf{B})$ numerical error, Part II of the companion paper [21] is devoted to extensive comparison of these variants. In yet another forthcoming paper, Part III, complex multiscale high speed turbulent astrophysical applications will be sought. Throughout the paper, the term " $\nabla \cdot \mathbf{B}$ numerical error" refers to the "amount of non-zero value of the discretized form of $\nabla \cdot \mathbf{B}$ of the underlying scheme".

\section{New Approach}

Aside from possessing the desirable property of adaptive numerical dissipation control of our proposed MHD scheme, the new approach consists of several additional new concepts. First, our filter scheme employs high order central spatial difference operators that preserve the divergence-free condition of the magnetic field as the base scheme. The only possible violation of the divergence-free condition is from the filter step. Second, a new idea in applying our filter procedure for minimizing the numerical error of $\nabla \cdot \mathbf{B}$ for high order schemes is proposed. For certain flows, we are able to preserve the divergence-free condition for the complete scheme. The third new concept is as follows:

The MHD equations are a system of non-strictly hyperbolic conservation laws. The non-convexity of the inviscid flux vector results in corresponding Jacobian matrices with undesirable properties. It has previously been shown by Powell et al. [10] that an "almost" equivalent MHD system in non-conservative form can be derived. In order to have a better conditioned eigensystem for the application of high-resolution shock-capturing schemes, they adjoined ad hoc non-conservative terms to the conservative equation. Actually, the MHD equations can be derived from basic principles in either conservative or non-conservative form (Marcel Vinokur of NASA Ames, private communication, 1996). 
We formulate our new scheme together with the Cargo \& Gallice [3] form of the MHD Roe approximate Riemann solver in curvilinear grids for both versions of the MHD equations. A novel feature of our new method is that the well-conditioned eigen-decomposition of the non-conservative MHD equations, with a minor modification, is used to solve the conservative equations. This new feature of the method provides well-conditioned eigenvectors for the conservative formulation, so that correct wave speeds for discontinuities are assured. Another advantage of using the non-conservative eigen-decomposition to solve the conservative equations is that our scheme exhibits smaller $\nabla \cdot \mathbf{B}$ numerical error. It will be shown that this approach, using the non-conservative eigensystem when solving the conservative equations, also works well in the context of standard shock-capturing schemes involving the use of the eigen-structure of the MHD equations.

\section{Description of the Scheme}

A full description of our adaptive low dissipative high order scheme for the Euler and Navier-Stokes equations can be found in $[18,19,12,20,15]$. Here, we describe the extension of this scheme to the MHD equations with the blending of high order nonlinear filters and high order linear filters. An important ingredient in our method is the use of the dissipative portion of high-resolution shock-capturing schemes as nonlinear filters. These nonlinear filters involve the use of approximate Riemann solvers. We will therefore first present a new form of high-resolution shock-capturing schemes for the conservative MHD equations using the non-conservative eigensystem.

\subsection{Preliminary}

Consider the 3-D non-conservative form of the ideal MHD equations

$$
\left(\begin{array}{c}
\rho \\
\rho u \\
\rho v \\
\rho w \\
e \\
B_{x} \\
B_{y} \\
B_{z}
\end{array}\right)_{t}+\operatorname{div}\left(\begin{array}{c}
\rho \mathbf{u u}^{T}+\left(p+\frac{1}{2} B^{2}\right) I-\mathbf{B B}^{T} \\
\mathbf{u}\left(e+p+\frac{1}{2} B^{2}\right)-\mathbf{B}\left(\mathbf{u}^{T} \mathbf{B}\right) \\
\mathbf{u} \mathbf{B}^{T}-\mathbf{B} \mathbf{u}^{T}
\end{array}\right)=-(\nabla \cdot \mathbf{B})\left(\begin{array}{c}
0 \\
B_{x} \\
B_{y} \\
B_{z} \\
\mathbf{u}^{T} \mathbf{B} \\
u \\
v \\
w
\end{array}\right)
$$

where the velocity vector is denoted $\mathbf{u}=(u, v, w)^{T}$, the magnetic field vector is $\mathbf{B}=$ $\left(B_{x}, B_{y}, B_{z}\right)^{T}, \rho$ is the density, and $e$ is the total energy. The vector on the right hand side is the non-conservative portion of the MHD equations. The notation $B^{2}=B_{x}^{2}+B_{y}^{2}+B_{z}^{2}$ is used. The pressure is related to the other variables by

$$
p=(\gamma-1)\left(e-\frac{1}{2} \rho\left(u^{2}+v^{2}+w^{2}\right)-\frac{1}{2}\left(B_{x}^{2}+B_{y}^{2}+B_{z}^{2}\right)\right)
$$

For plasmas, $\gamma$ is usually equal to 1.667 (for Mon-atomic gases).

We will also consider the conservative MHD equations, obtained by setting the right hand side equal to zero. The non-conservative term is proportional to the divergence of the magnetic field $(\nabla \cdot \mathbf{B})$. Physically, it is zero if $\nabla \cdot \mathbf{B}=0$ initially.

In symbolic form, the non-conservative form can be written as 


$$
U_{t}+\nabla \cdot F=S
$$

where $U$ is the corresponding state vector, $F$ is the conservative inviscid flux vector and $S$ is the non-conservative portion of the equations in (1).

There has been much concern in preserving the divergence-free condition throughout the computation, see e.g., $[5,16]$ and references cited therein. The $\nabla \cdot \mathbf{B}=0$ condition is an initial constraint for the MHD equations. The divergence-free condition is not part of the MHD differential system, and it is very different from the divergence-free condition for the incompressible Euler or Navier-Stokes equations. The divergence-free condition for the incompressible Euler or Navier-Stokes is part of the differential system. It is needed to close the system and must be enforced explicitly. For the MHD equations, as long as the magnitude of the discretized form of $\nabla \cdot \mathrm{B}$ is on the order of truncation error, and goes to zero when the grid is refined, there should be no problem. Unfortunately, not all schemes preserve the divergence-free condition. This is especially true for standard upwind and high-resolution shock-capturing schemes that involve some form of Riemann solver.

When using pure centered difference operators, it is trivial to see that the divergence of $\mathrm{B}$ is perfectly preserved. Take for example the semi-discrete approximation of the magnetic field equations

$$
\begin{aligned}
& \frac{d B_{x}(t)_{i, j, k}}{d t}+ \\
& D_{j}\left(B_{y_{i, j, k}} u_{i, j, k}-B_{x i, j, k} v_{i, j, k}\right)+D_{k}\left(B_{z i, j, k} u_{i, j, k}-B_{x i, j, k} w_{i, j, k}\right)=0 \\
& \frac{d B_{y}(t)_{i, j, k}}{d t}+ \\
& \frac{d B_{\boldsymbol{z}}(t)_{i, j, k}}{d t}+ \\
& D_{i}\left(B_{x i, j, k} v_{i, j, k}-B_{y_{i, j, k}} u_{i, j, k}\right)+D_{k}\left(B_{z i, j, k} v_{i, j, k}-B_{y_{i, j, k}} w_{i, j, k}\right)=0 \\
& D_{i}\left(B_{x i, j, k} w_{i, j, k}-B_{z i, j, k} u_{i, j, k}\right)+D_{j}\left(B_{y_{i, j, k}} w_{i, j, k}-B_{z i, j, k} v_{i, j, k}\right)=0,
\end{aligned}
$$

where $D_{i}, D_{j}$ and $D_{k}$ denote finite difference operators acting in the $i$-, $j-, k$-directions, respectively.

Forming the divergence by taking the sum of $D_{i}$ on the $B_{x}$ equation, $D_{j}$ on the $B_{y}$ equation, and $D_{k}$ on the $B_{z}$ equation gives

$$
\frac{d\left(D_{i} B_{x}+D_{j} B_{y}+D_{k} B_{z}\right)_{i, j, k}}{d t}=-D_{i} D_{j} B_{y_{i, j, k}} u_{i, j, k}+D_{j} D_{i} B_{y_{i, j, k}} u_{i, j, k}+\ldots=0,
$$

where the dots denote several additional terms of the similar form as the first two. All these terms disappear, since the difference operators along different coordinate directions commute, i.e., $D_{i} D_{j}=D_{j} D_{i}$.

When the solution is smooth, we can use a high order centered difference operator and perfectly preserve the divergence-free condition. In this case the result will be the same, whether we solve the conservative or non-conservative equations.

From now on, the discussion pertains to schemes involving the use of Riemann solvers or the eigen-structure of the MHD equations. For convenience of presentation, we will describe our numerical methods for 1-D problems on a uniform grid. The 1-D MHD equations become

$$
\begin{gathered}
U_{t}+F(U)_{x}=0 \quad \text { (conservative) } \\
U_{t}+F(U)_{x}=S \quad \text { (non-conservative) }
\end{gathered}
$$


Let $A(U)$ denote the Jacobian $\partial F / \partial U$ with the understanding that the present $U, F$ and $S$ are the 1-D counterpart of the 3-D description above. For later discussion, we write the non-conservative terms $S=N(U) U_{x}$.

Presently, there are basically two camps in solving the MHD equations; namely, solving the conservative or more recently, popularized by [10], the non-conservative form. In both cases high-resolution shock capturing methods suffer from the need to perform extra work to keep the $\nabla \cdot \mathbf{B}$ numerical error to machine zero. Three of the popular approaches are to minimize the $\nabla \cdot \mathbf{B}$ numerical error by augmenting an extra PDE to the system [5], using the staggered approach of K.S. Yee [22, 6, 7], and a projection method [11]. There is, however, a key advantage to solving the conservative equations over the non-conservative equations since the conservative form guarantees correct propagation speeds and locations of discontinuities.

It can be shown that the "non-conservative Jacobian" $A(U)-N(U)$ has real eigenvalues, and a complete set of eigenvectors [3]. The conservative Jacobian $A(U)$ has real eigenvalues, but there exist states $U$ for which $A(U)$ does not have a complete set of eigenvectors. Therefore, defining an upwind scheme based on $A(U)$ is problematic.

In the next subsection, we show a simple way of constructing upwind or high-resolution shock-capturing schemes that use approximate Riemann solvers for the conservative equations without the use of its ill-conditioned conservative eigensystem. This will set the stage for a conservative filter approach involving the use of the resulting nonlinear dissipative portion of these high-resolution shock-capturing schemes.

\subsection{Conservative and Non-Conservative Formulations Involving the Use of Approximate Riemann Solvers}

For simplicity of presentation, we illustrate the idea for a first-order upwind scheme involving the use of the MHD counterpart of Roe's approximate Riemann solver for the gas dynamic equations. Here we show that the construction of an upwind scheme for the conservative equations is possible by using the non-conservative eigen-decomposition. Furthermore, it will be shown by numerical experiments that the conservative method will give smaller generation of spurious divergence of the magnetic field, than that obtained when solving the non-conservative equations.

In Cargo \& Gallice [3] a matrix, $A_{j+1 / 2}=A\left(U_{j}, U_{j+1}\right)$, consistent with the Jacobian, and having the "property $U$ " of Roe for the MHD equations

$$
F_{j+1}-F_{j}=A_{j+1 / 2}\left(U_{j+1}-U_{j}\right)
$$

is constructed. Unlike the case of standard gas dynamics, this matrix cannot be written as the Jacobian at an average state of $A$. By inspecting terms in the matrix, they use the decomposition $A_{j+1 / 2}=\left(A_{j+1 / 2}-N_{j+1 / 2}\right)+N_{j+1 / 2}$, where $N_{j+1 / 2}$ is consistent with $N(U)$. This is due to the fact that there is a simple relationship between the eigenstructure of the conservative and the non-conservative forms. Here $N(U)$ is part of the non-conservative terms $S=N(U) U_{x}$.

It turns out that seven of the eigenvalues and eigenvectors are identical for the Jacobian matrices $\left(A_{j+1 / 2}\right)$ and $\left(A_{j+1 / 2}-N_{j+1 / 2}\right)$ [8]. Assuming the eigenvalues are arranged in the increasing order, the difference between the two matrices lies only in the eighth eigenvector. 
Furthermore, the eighth eigenvector of the conservative system can sometimes coincide with one of the eigenvectors, thereby, making it impossible to define the MHD Roe's approximate Riemann solver in the standard way. The eigenvectors of the non-conservative Jacobian $\left(A_{j+1 / 2}-N_{j+1 / 2}\right)$ are very simple.

We proposed to use the non-conservative form but with the fourth eigenvalue (the degenerate eigenvalue) replaced by zero for the conservative form, since only the eighth eigenvector of the non-conservative form is not the same as the eighth eigenvector for the conservative form. The incorrect eigenvector will be multiplied by an eigenvalue which is close to zero (the eigenvalue will not be exactly zero when an entropy correction is used; see below). Thus the effect of a "false" eigenvector will be small. By using the eighth eigenvector of the non-conservative system instead, the difficulty will be avoided.

Let $\mu_{j+1 / 2}^{k}, k=1, \ldots, 8$ denote the eigenvalues of the conservative Jacobian $A_{j+1 / 2}$. Let $\lambda_{j+1 / 2}^{k}$ and $\mathrm{r}_{j+1 / 2}^{k}$ denote the eigenvalues and eigenvectors, respectively, of the nonconservative Jacobian $A_{j+1 / 2}-N_{j+1 / 2}$. With the above justification, we define the numerical upwind flux of the conservative equations as

$$
H_{j+1 / 2}^{c}=\frac{1}{2}\left(F_{j+1}+F_{j}\right)-\frac{1}{2} \sum_{k=1}^{8} q\left(\mu_{j+1 / 2}^{k}, \epsilon\right) \alpha_{j+1 / 2}^{k} \tilde{\mathbf{r}}_{j+1 / 2}^{k},
$$

where $\alpha_{j+1 / 2}^{k}$ is the jump of the respective characteristic variables of $U_{j+1}-U_{j}$ in the eigenvector basis, and $q(x, \epsilon)$ is the entropy-corrected absolute value, e.g., $q(x, \epsilon)=\max (|x|, \epsilon)$, where $\epsilon$ is a small parameter. Here $\tilde{r}_{j+1 / 2}^{k}$ is the minor modification of non-conservative eigenvectors discussed previously (only the eighth eigenvector is modified).

For the non-conservative equations, we define the corresponding numerical flux

$$
H_{j+1 / 2}^{n c}=\frac{1}{2}\left(F_{j+1}+F_{j}\right)-\frac{1}{2} \sum_{k=1}^{8} q\left(\lambda_{j+1 / 2}^{k}, \epsilon\right) \alpha_{j+1 / 2}^{k} \mathbf{r}_{j+1 / 2}^{k} .
$$

Making use of the identity $A_{j+1 / 2}=\left(A_{j+1 / 2}-N_{j+1 / 2}\right)+N_{j+1 / 2}$, the semi-discrete nonconservative upwind approximation of the MHD equations can be written as

$$
\frac{d U_{j}}{d t}=-\frac{1}{\Delta x}\left(H_{j+1 / 2}^{n c}-H_{j-1 / 2}^{n c}\right)+\frac{1}{2 \Delta x}\left(N_{j+1 / 2}\left(U_{j+1}-U_{j}\right)+N_{j-1 / 2}\left(U_{j}-U_{j-1}\right)\right) .
$$

The semi-discrete conservative upwind approximation of the MHD equations is the usual form

$$
\frac{d U_{j}}{d t}=-\frac{1}{\Delta x}\left(H_{j+1 / 2}^{c}-H_{j-1 / 2}^{c}\right)
$$

The conservative and non-conservative TVD and WENO schemes used in this paper are generalized from either of these two first-order schemes in a standard way.

\subsection{High Order ACM and Wavelet NonLinear/Linear Filter Method}

Our high order filter method consists of two stages, a divergence-free preserving base scheme stage and a filter stage which can be divergence-free preserving depending on the type of filter operator being used and the method of applying the filter step. 
Divergence-Free Preserving Base Schemes: The first stage of the numerical method consists of a time step by a spatially high order non-dissipative and high order temporally base scheme operator $L$ (e.g., a divergence-free preserving sixth-order central in space and high order linear-multistep or fourth-order Runge-Kutta in time),

$$
U^{*}=L\left(U^{n}\right)
$$

where $U^{n}$ is the numerical solution vector at time level $n$. When necessary, a high order linear numerical dissipation operator can be used. For example, a divergence-free preserving eighth-order linear dissipation with the sixth-order centered base scheme to approximate $F(U)_{x}$ is written, in the 1-D case, as

$$
\frac{\partial F}{\partial x} \approx-D_{06} F_{j}-d \Delta x^{7}\left(D_{+} D_{-}\right)^{4} U_{j}
$$

where $D_{06}$ is the standard sixth-order accurate centered difference operator, and $D_{+} D_{-}$is the standard second-order accurate centered approximation of the second derivative. $d$ is a tunable parameter. The operators are modified at boundaries in a stable way [12]. (Note that for more than two level linear-multistep methods, the $L$ operator involves the corresponding number of time levels.) This highly accurate base scheme is constructed to numerically preserve the divergence-free condition of the magnetic field (to the level of round-off error) for curvilinear grids. The only possible source of violation of the divergence-free condition is from the filter step (assuming the correct handling of the physical and numerical boundary conditions).

Adaptive Numerical Dissipation Filters: After the completion of a full time step of the divergence-free preserving base scheme, the second stage is to filter the solution by the dissipative portion of a high-resolution shock-capturing scheme. The filter operator can be written as (assume 1-D for ease of illustration)

$$
U_{j}^{n+1}=U_{j}^{*}-\frac{\Delta t}{\Delta x}\left[H_{j+1 / 2}^{f}-H_{j-1 / 2}^{f}\right] .
$$

The filter numerical flux vector is

$$
H_{j+1 / 2}^{f}=R_{j+1 / 2} \bar{H}_{j+1 / 2} .
$$

Here $R_{j+1 / 2}$ is the matrix of right eigenvectors of the Jacobian of the non-conservative MHD flux vector $\left(A_{j+1 / 2}-N_{j+1 / 2}\right)$ as discussed in the previous subsection. The $\bar{H}_{j+1 / 2}$ are also evaluated from the same characteristic quantities derived from these eigenvectors. Denote the elements of the vector $\bar{H}_{j+1 / 2}$ by $\bar{h}_{j+1 / 2}^{l}, l=1,2, \ldots, 8$. They have the form

$$
\bar{h}_{j+1 / 2}^{l}=\left(s^{N}\right)_{j+1 / 2}^{l}\left(\phi_{j+1 / 2}^{l}\right)-\left(s^{L}\right)_{j+1 / 2}^{l} d_{j+1 / 2}^{l} .
$$

Here $\left(s^{N}\right)_{j+1 / 2}^{l}$ and $\left(s^{L}\right)_{j+1 / 2}^{l}$ are sensors to activate the higher order nonlinear filter and linear filter, respectively. For example, $\left(s^{N}\right)_{j+1 / 2}^{l}$ is designed to be zero in regions of smooth flow and near one in region with discontinuities. $\left(s^{N}\right)_{j+1 / 2}^{l}$ varies from one grid point to another and is obtained either from a wavelet analysis of the solution (WAV-filter scheme), or from a gradient-based detector (ACM-filter scheme) $[18,19,12,20,15]$. We have in the previous numerical experiments [20] used $\left(s^{L}\right)_{j+1 / 2}^{l}=1-\left(s^{N}\right)_{j+1 / 2}^{l}$, but other choices are possible. See [20] for a discussion. The functions $\phi_{j+1 / 2}^{l}$ and $d_{j+1 / 2}^{l}$ are the nonlinear and linear filters for the $l$ th-characteristic wave. 
The nonlinear filter $\phi_{j+1 / 2}^{l}=g_{j+1 / 2}^{l}-b_{j+1 / 2}^{l}$ is the dissipative portion of a uniformly high order high-resolution shock-capturing scheme for the $l$ th-characteristic wave. Here $g_{j+1 / 2}^{l}$ and $b_{j+1 / 2}^{l}$ are numerical fluxes of the uniformly high order high-resolution scheme and a high order central scheme for the $l$ th characteristic, respectively. It is noted that $b_{j+1 / 2}^{l}$ might not be unique since there is more than one way of obtaining $\phi_{j+1 / 2}^{l}$. For the forms of the $\phi_{j+1 / 2}^{l}$ used in the numerical experiment section, see $[18,19,12,20,15]$. For example, the form of Harten and Yee and symmetric TVD schemes are already in the proper form in the sense that they are written in a central differencing portion $b_{j+1 / 2}^{l}$ and a nonlinear dissipation portion $\phi_{j+1 / 2}^{l}$. No work is required to obtain $\phi_{j+1 / 2}^{l}$ in this case.

The linear filter $d_{j+1 / 2}^{l}$ consists of a tuning parameter $d_{f}$. For a sixth-order spatial base scheme, the eighth-order central dissipation for the linear filter is used. For the test cases to be shown later, the majority of the computations only use the nonlinear filter. It is not necessary to use the linear filter, i.e., setting $\left(s^{L}\right)_{j+1 / 2}^{l}=0$.

When Runge-Kutta time stepping is used, the filter is usually applied after the completion of each full time step, or for extremely high speed flows, applied after each stage in the Runge-Kutta method. In all of the numerical examples shown later, we filter the solutions after the completion of each full Runge-Kutta time step.

Options in Filtering the Magnetic Field Equations: In order to minimize the numerical error of the divergence-free magnetic condition, the nonlinear filter step only acts on the hydrodynamic portion of the equations. To further improve nonlinear stability and accuracy, if necessary, we might employ high order linear filters that preserve the divergence-free property for the magnetic field portion of the equations. For problems containing moderate to strong shocks that are magnetically dominated, it might be necessary to apply a nonlinear filter on the magnetic field equations as well. In this case, the scheme, through wavelet sensors, still maintains the divergence-free preserving property in smooth regions of the flow. Construction of accurate schemes for magnetically dominated shocks is a subject of ongoing research. The next section illustrates the representative property of our scheme for mainly shock wave-dominated MHD test cases.

\section{Shock Wave Dominated MHD Numerical Examples}

For illustration purposes, numerical examples using sixth-order central spatial and fourthorder temporal discretizations as the base scheme with adaptive numerical dissipation controls for four typical MHD model problems are included. These are shock wave-dominated examples. The purpose is to validate our new scheme for conventional MHD model problems. Since most of these examples are not turbulence/combustion flows, the full capability of the new scheme is not utilized. Thus, we do not expect the new scheme to exhibit noticeable improvement in stability and shock-resolution over conventional schemes. In a forthcoming paper, multiscale MHD examples will be included and new features of the present scheme will surface.

The sixth-order base scheme together with the nonlinear/linear filter with wavelet sensor will be denoted WAV66. When a more conventional gradient based sensor is used, the scheme is denoted ACM66. If high order linear numerical dissipation is also used in the base scheme, the methods will be denoted WAV66+AD8 and ACM66+AD8 respectively. 
The strength of the eighth-order dissipation will be denoted by a tunable coefficient $d$, as in (4). When a high order filter is used, its dissipation strength will be denoted by $d_{f}$.

For comparison, the fifth-order weighted ENO scheme (denoted WENO5), described in [9] will also be used. Classical fourth-order Runge-Kutta time stepping is used for all sixthorder schemes, as well as for the WENO5 scheme. The second-order TVD schemes HartenYee and MUSCL are integrated in time by the second-order TVD Runge-Kutta method. The conservative (3) and non-conservative (2) versions of the TVD schemes have both been implemented and will be compared below. All the methods use the same approximate Riemann solver of Cargo \& Gallice. Unless indicated, all of the computations use uniform grids and solve the conservative form of the MHD equations with the non-conservative eigenvector decomposition as discussed in Section 4.2, and Section 4.3 for the filter scheme (ACM66 and WAV66). In addition, for ACM66, ACM66+AD8, WAV66 and WAV66+AD8, unless indicated, the nonlinear filter are applied on the full MHD system on numerical solutions shown.

The $\nabla \cdot \mathrm{B}$ numerical error is obtained by approximating the spatial derivatives by sixthorder centered differences for WAV66, ACM66 and WENO5, whereas the corresponding $\nabla \cdot \mathbf{B}$ numerical error is obtained by second-order centered differences for the second-order TVD schemes (MUSCL and Harten-Yee).

\subsection{A 1-D Riemann Problem}

Here we consider the same 1-D Riemann (shock-tube) problem as examined by Brio \& Wu [2]. This Riemann problem is chosen such that the $x$ and $z$ components of the magnetic field, $B_{x}$ and $B_{z}$, are constant throughout the time evolution. In other words, the magnetic field does not change direction, only magnitude. The initial data for the left $(L)$ and right (R) states are

$$
\left(\begin{array}{c}
\rho \\
\rho u \\
\rho v \\
\rho w \\
e \\
B_{x} \\
B_{y} \\
B_{z}
\end{array}\right)_{L}=\left(\begin{array}{c}
1 \\
0 \\
0 \\
0 \\
2.28125 \\
0.75 \\
1 \\
0
\end{array}\right), \quad\left(\begin{array}{c}
\rho \\
\rho u \\
\rho v \\
\rho w \\
e \\
B_{x} \\
B_{y} \\
B_{z}
\end{array}\right)_{R}=\left(\begin{array}{c}
0.125 \\
0 \\
0 \\
0 \\
0.93125 \\
0.75 \\
-1 \\
0
\end{array}\right)
$$

where $\rho$ is the density, $u, v$ and $w$ are the velocity components, and $e$ is the total energy per unit volume. The $B_{x}, B_{y}$ and $B_{z}$ are the three magnetic field components. The density and the $y$-component of the magnetic field of a reference solution obtained by a second-order Harten-Yee TVD scheme for the non-conservative MHD form of the equations, using 16000 uniform grid points, are shown in Fig. 1. Note that the eighth wave is not present and the rotational discontinuities are also not present in this 1-D co-planar Riemann problem. We are thus left with 5 waves.

Comparisons of MUSCL, Harten-Yee and WENO5 with the present scheme were conducted to validate our approach. All computations were run on the domain $0<x<1$ to time 0.12 , using a fixed CFL number of 0.2 and using the same non-conservative form of the MHD equations. A uniform grid of 400 points is used for the comparison. The value of the gas law parameter $\gamma$ was 1.6667. Figure 2 shows the density using the MUSCL second-order TVD scheme with the van Albada limiter (solid: reference solution; circle: 

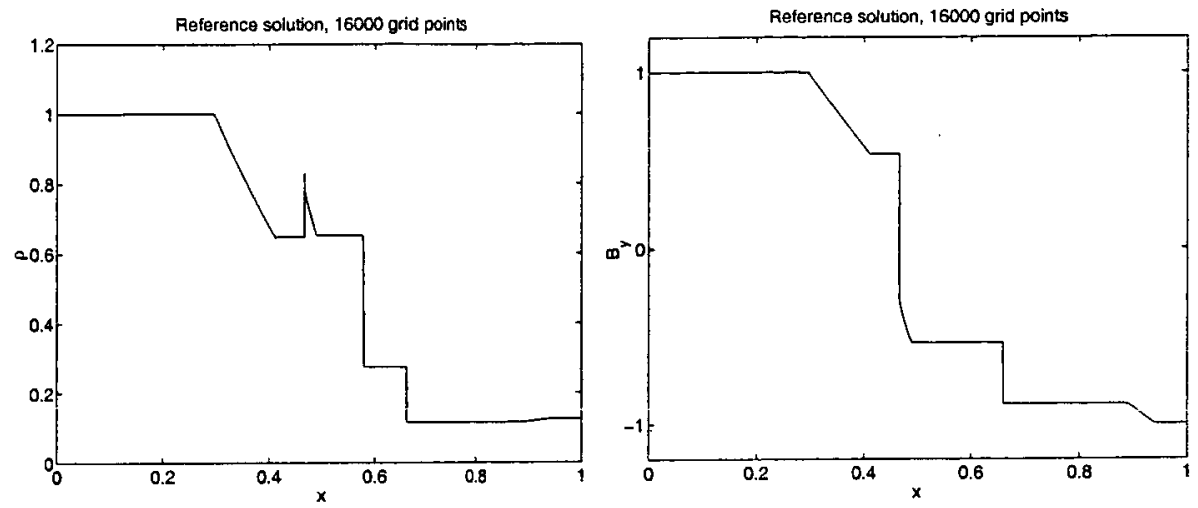

Fig. 1. Reference Solution for the 1-D Riemann problem. Density and $y$-component of the magnetic field computed by Harten-Yee at time 0.12 using a uniform 16000 grid.
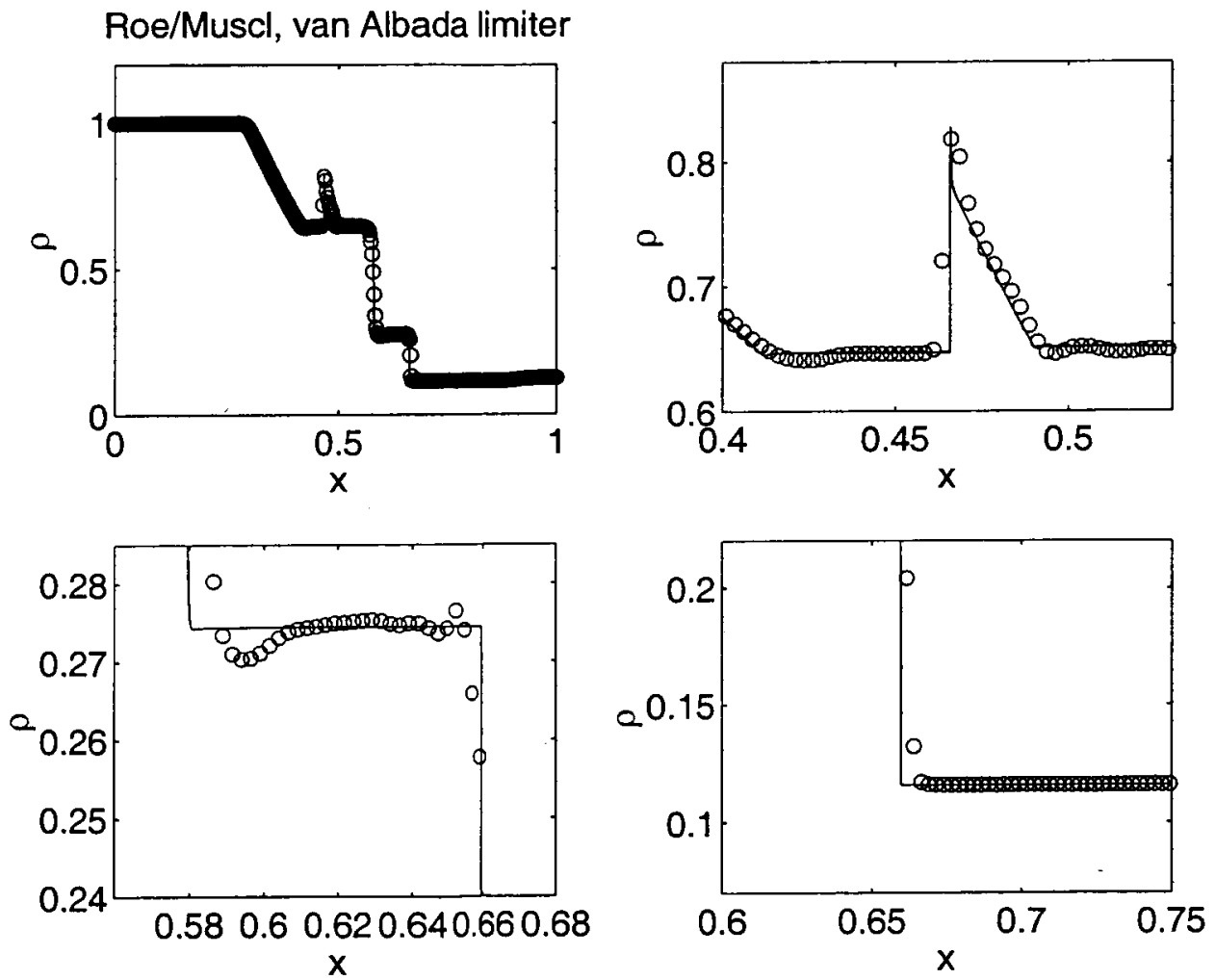

Fig. 2. 1-D Riemann problem. Density computed by MUSCL with van Albada limiter using a uniform 400 grid. 

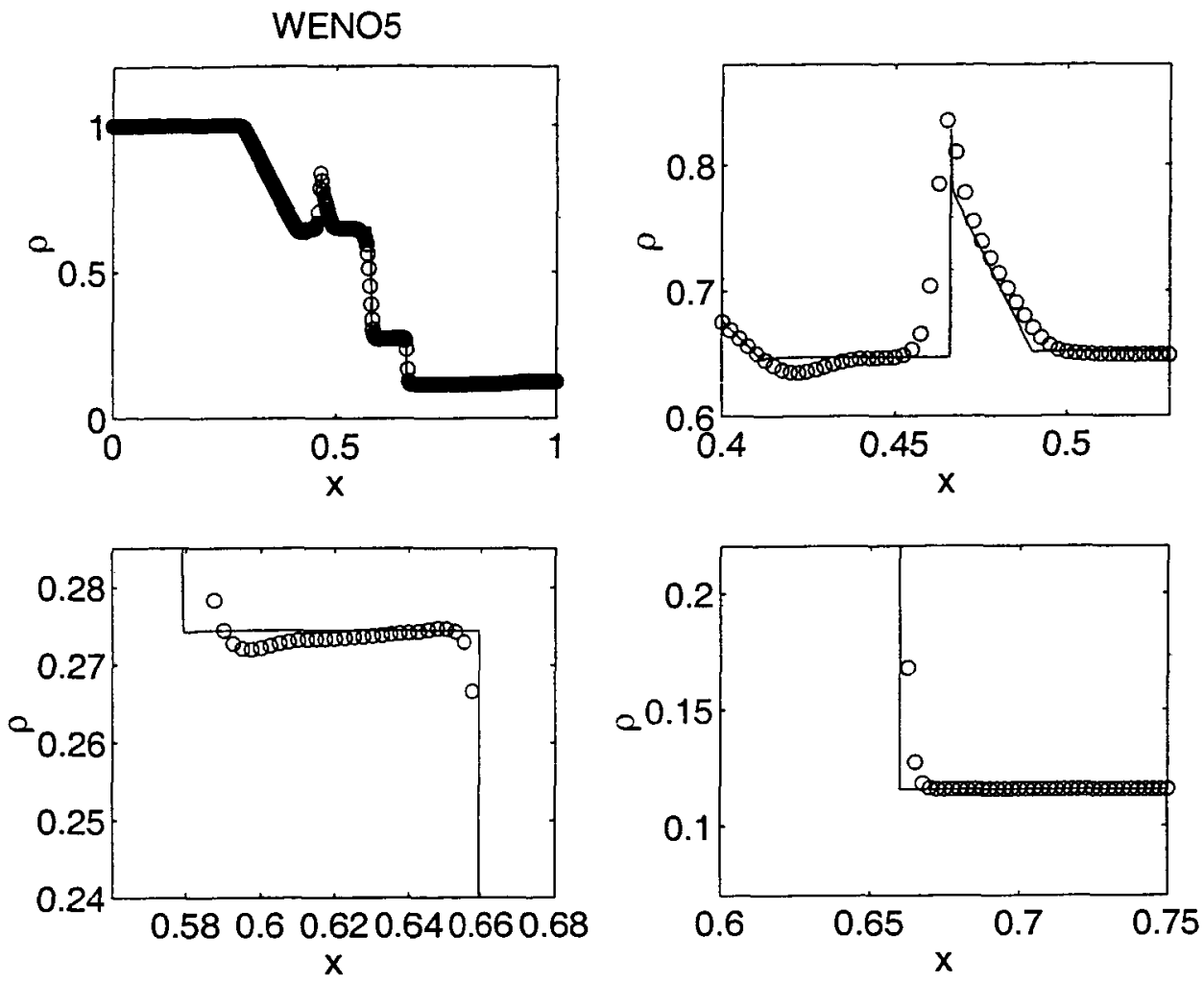

Fig. 3. 1-D Riemann problem. Density computed by WENO5 using a uniform 400 grid.

MUSCL). The upper left plot shows the solution on the entire computational domain. The sub-figures zoom in on different key parts of the domain.

Figure 3 shows the computation by WENO5. Figure 4 shows the computation using the WAV66+AD8 filter scheme (sixth-order spatial base scheme, second-order nonlinear filter and blending with a small amount of high order linear filter). It can be seen that the WAV66+AD8 exhibits a slightly higher accuracy than the MUSCL and WENO5 schemes. Note that for this rapidly developing short time integration flow without any structure away from discontinuities, it is expected that any good second-order shockcapturing scheme would perform well. The small over- and undershoots are characteristic of all studied schemes (and numerical results published in the open literature) even with the use of 8000 uniform grid points. Adaptive grid refinement or the use of extremely fine grids (e.g., 16000 grid points) is necessary to minimize the small oscillations.

\subsection{Orszag-Tang Vortex}

The purpose of this example is to illustrate the advantage of the filter approach over the standard shock-capturing scheme in minimizing the numerical error of the divergence-free magnetic condition. The advantage of the present conservative scheme over the conventional conservative scheme is also illustrated.

The 2-D Orszag-Tang vortex problem consists of periodic boundary conditions with smooth initial data 
WAV66, van Albada $\varepsilon=0.2, \mathrm{ds}=0.0001, \mathrm{df}=0.002$
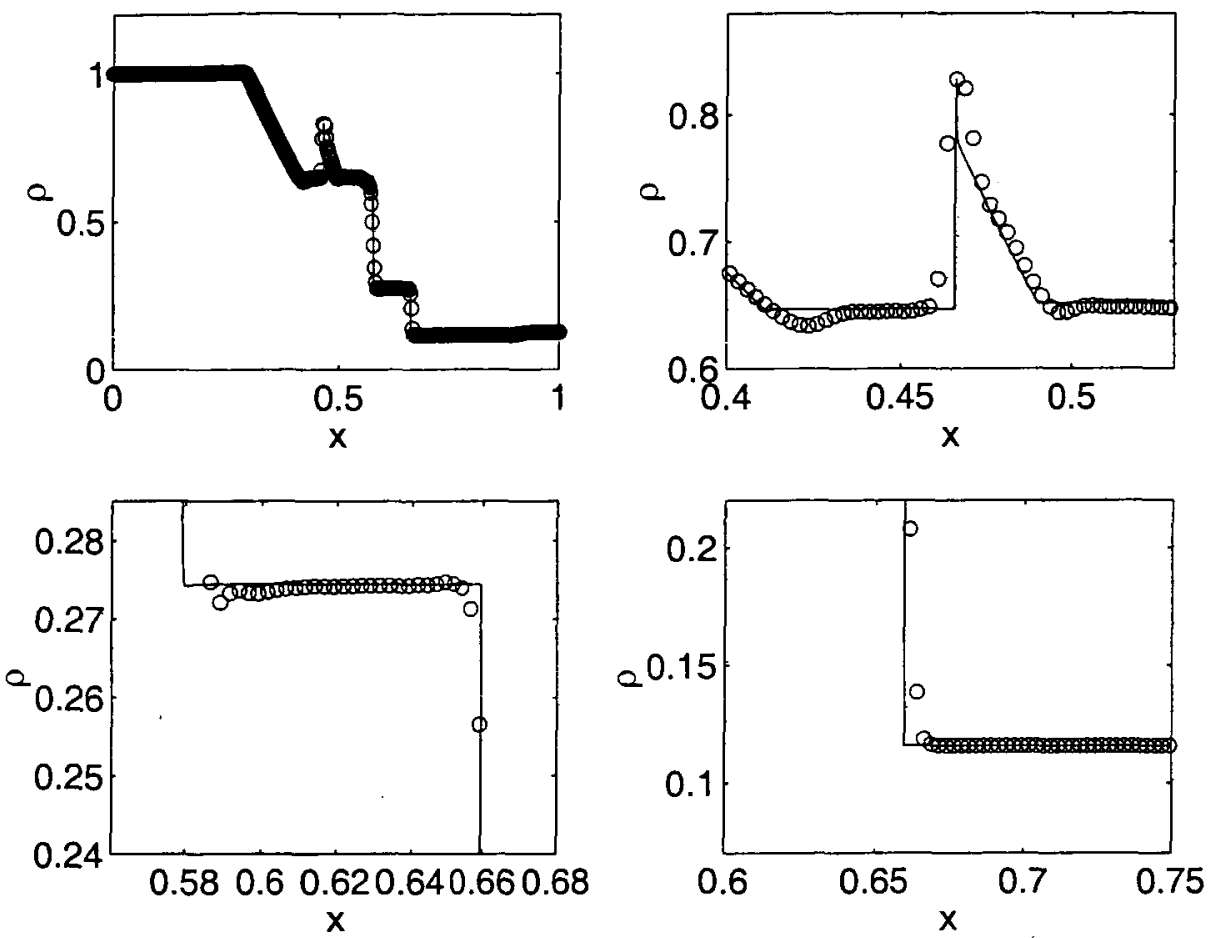

Fig. 4. 1-D Riemann problem. Density computed by WAV66+AD8 with van Albada limiter using a uniform 400 grid.

$$
\left(\begin{array}{c}
\rho \\
u \\
v \\
w \\
p \\
B_{x} \\
B_{y} \\
B_{z}
\end{array}\right)=\left(\begin{array}{c}
25 / 9 \\
-\sin y \\
\sin x \\
0 \\
5 / 3 \\
-\sin y \\
\sin 2 x \\
0
\end{array}\right)
$$

The computational domain is $0<x<2 \pi, 0<y<2 \pi$. The computation stops at time $\mathrm{T}=3.14(\approx \pi)$ using a CFL number of 0.6 .

Density contours of the solution for three levels of grid refinement $(201 \times 201,401 \times 401$, and $801 \times 801$ ) by the Harten-Yee scheme solving the non-conservative MHD equations are displayed in Fig. 5. A grid convergence study of the WENO5 scheme, integrated in time with the classical fourth-order accurate Runge-Kutta method is shown in Fig. 6. The Harten-Yee scheme and the WENO5 schemes do not perfectly conserve the divergence of $B$. Here $B$ is the 3-D magnetic field vector. We expect a higher order method to give a smaller error in the $\nabla \cdot \mathbf{B}$. The development of the $L^{2}$ norm of $\nabla \cdot \mathbf{B}$ in time for the Harten-Yee scheme and the WENO5 scheme using $801 \times 801$ uniform grid points is shown in Fig. 7. As long as the solution is smooth, it is clear that a more accurate method gives a smaller increase in $\nabla \cdot \mathbf{B}$. However, when shocks have formed, the error is due to the 

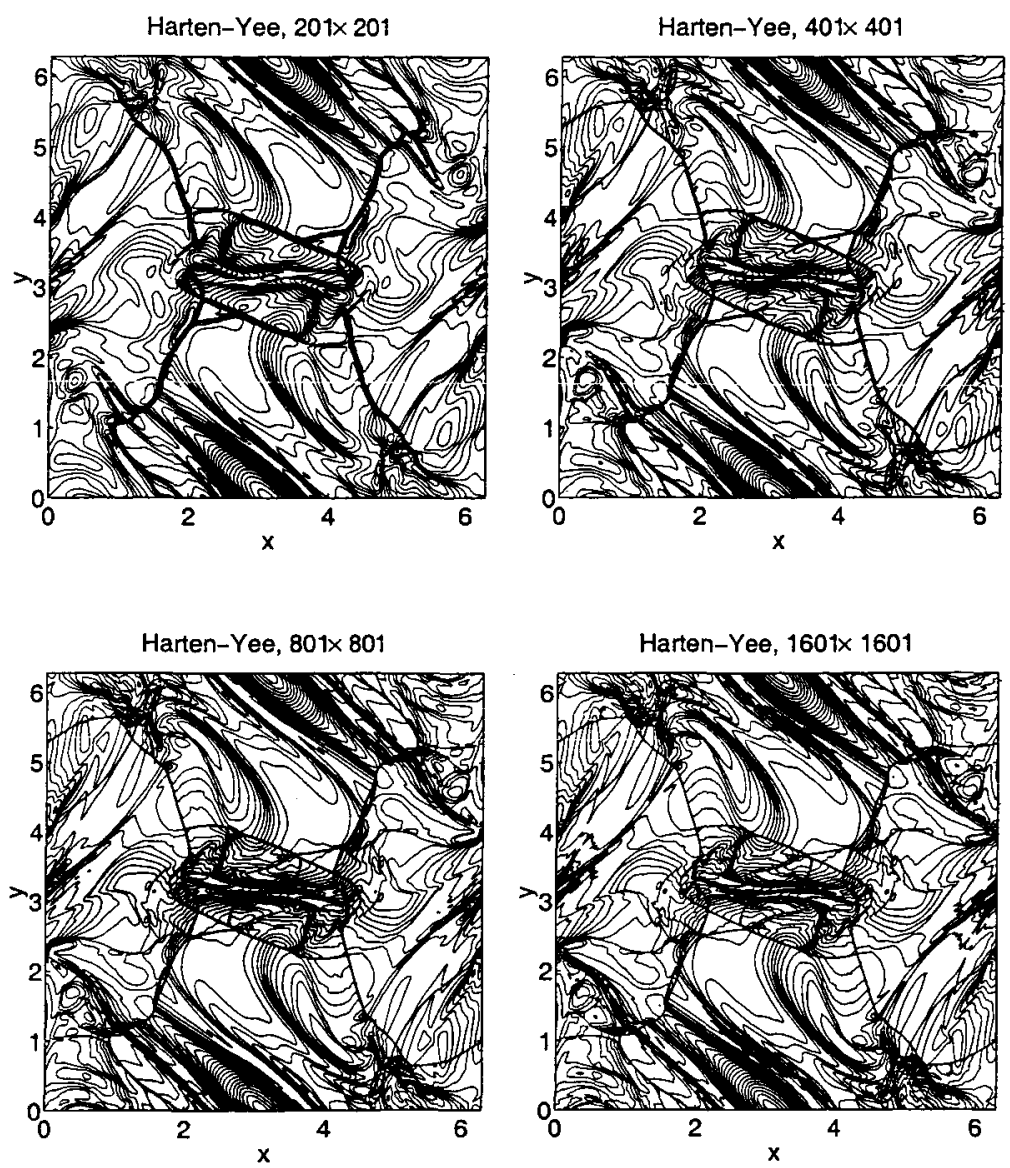

Fig. 5. Density contours: Grid refinement of the Orszag-Tang problem by Harten-Yee

amount of the nonlinear dissipation of the scheme in the vicinity of discontinuities. This is clearly seen in Fig. 7.

The $L^{2}$ norm of the numerical $\nabla \cdot \mathbf{B}$ as a function of time is shown in Fig. 9 for the three levels of grid refinement. It is clear that the norm of the divergence of the magnetic field does not converge to zero as the grid is refined. The errors in $\nabla \cdot \mathbf{B}$ are associated with shock formation, and should not be expected to diminish, since, unlike our filter approach, the Harten-Yee scheme is applied to the entire MHD equations. Figure 8 shows the computation by WAV66+AD8 using uniform grids of sizes $101 \times 101,201 \times 201$, and $401 \times 401$.

Figure 10 shows the $L^{2}$ norm of $\nabla \cdot \mathbf{B}$ for the Harten-Yee scheme (dashed curve) and the WAV66 scheme (solid curve) for the non-conservative form of the equations. Perfect preservation of the divergence-free condition is obtained by the WAV66 before shock waves develop. This is clearly visible in Fig. 10. Up to time around 0.7, the solution is smooth enough that the nonlinear filter is not very active. The norm of the $\nabla \cdot \mathbf{B}$ numerical error is then on the level of round-off error. This is due to the fact that, although the spatially sixth-order central base scheme of WAV66 preserves the divergence-free condition, the 

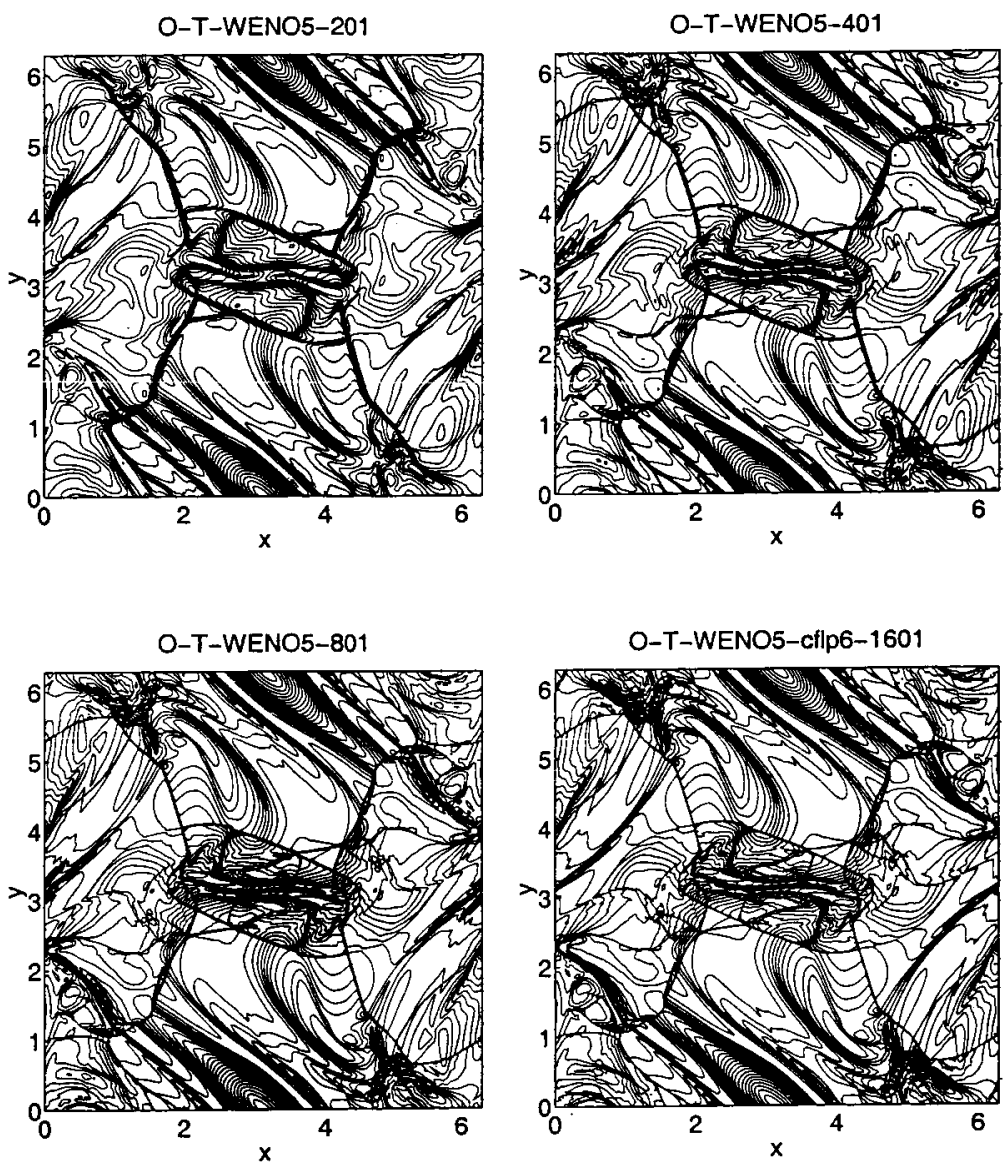

Fig. 6. Density contours: Grid refinement of the Orszag-Tang problem by WENO5

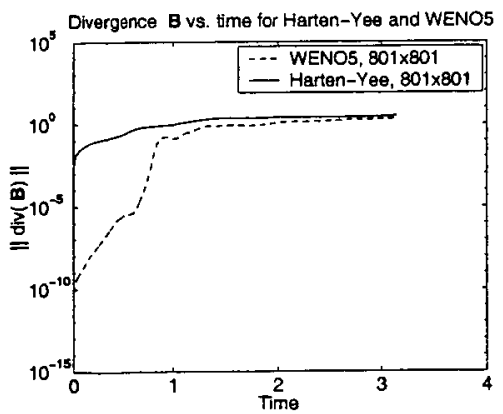

Fig. 7. Orszag-Tang problem. Divergence B versus time by WENO5 (dashed) and Harten-Yee (solid) using an uniform $801 \times 801$ grid. 

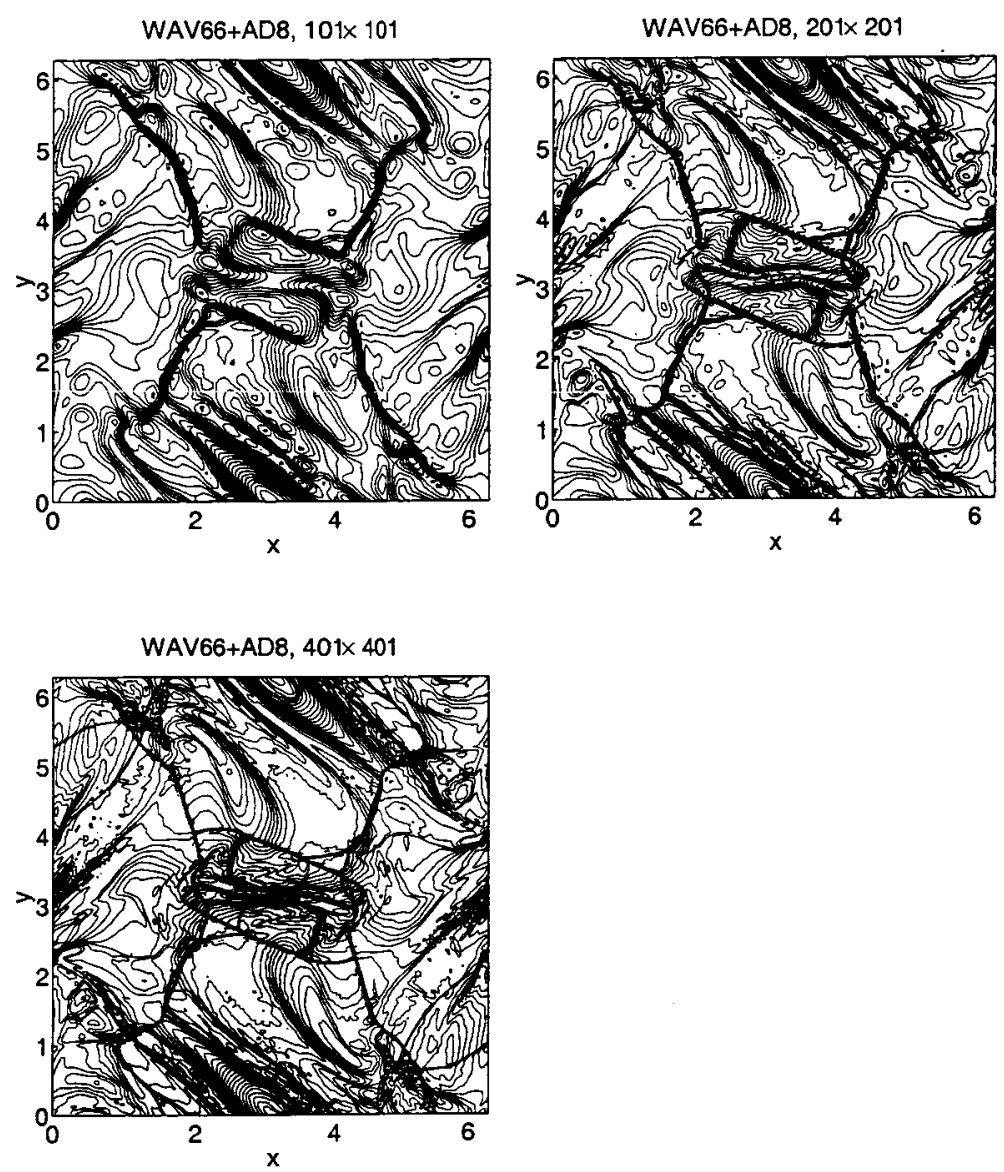

Fig. 8. Grid refinement study for the Orszag-Tang problem. Density contours by WAV66+AD8, $d=0.005$.

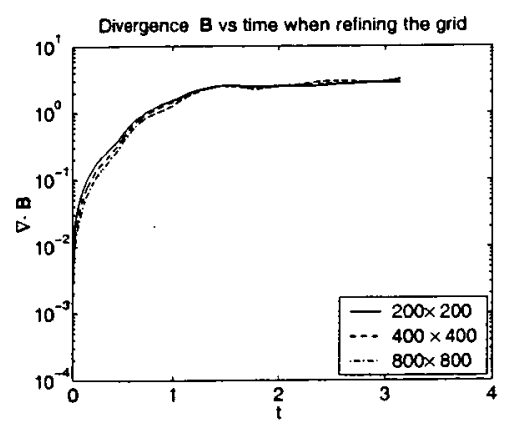

Fig. 9. Orszag-Tang problem. $L^{2}$ norm of numerical $\nabla \cdot \mathbf{B}$ vs. time on grids of increasing refinement by Harten-Yee. 


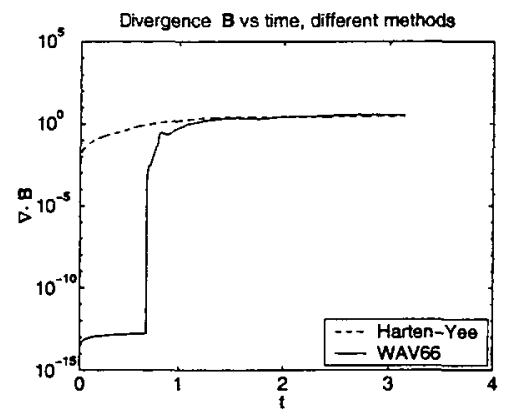

Fig. 10. Orszag-Tang problem. $L^{2}$ norm of numerical $\nabla \cdot \mathbf{B}$ vs. time. by WAV66 and Harten-Yee.

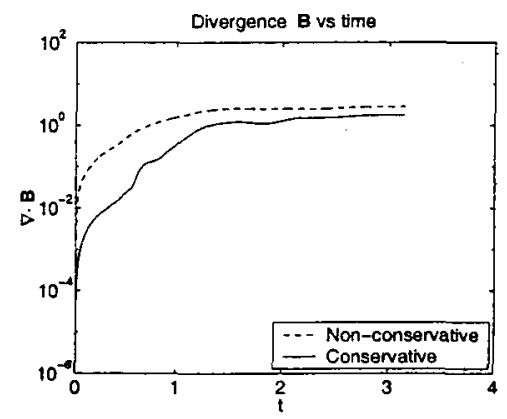

Fig. 11. Orszag-Tang problem. $L^{2}$ norm of numerical $\nabla \cdot \mathrm{B}$ vs. time. Conservative and nonconservative MHD by Harten-Yee.

nonlinear filter, even though not too active, introduces a numerical error in the $\nabla \cdot \mathbf{B}$ of the same size as the truncation error of the approximation. At later times the nonlinear filter dissipation of WAV66 quickly takes the $\nabla \cdot \mathbf{B}$ numerical value to that of the HartenYee scheme after the onset of shock waves.

A comparison of numerical results using MUSCL, ACM66 and ACM66+AD8 were also conducted. Since the accuracy of the numerical solution by the MUSCL method was inferior to the rest of the methods (as for all test cases), we will not discuss the MUSCL scheme from here on. The ACM66 and ACM66+AD8 are slightly more stable but exhibit a slightly larger $\nabla \cdot \mathbf{B}$ numerical error than WAV66 (figures not shown).

The development of the norm of the numerical $\nabla \cdot \mathbf{B}$ in time is shown in Fig. 11 for the conservative and the non-conservative forms of the MHD equations. The computations use the same Harten-Yee scheme and the same eigen-decomposition of the non-conservative equations to solve both forms of the MHD equations. The conservative form leads to a smaller $\nabla \cdot \mathbf{B}$ numerical error. The same result, that the conservative form of the MHD system gives smaller spurious $\nabla \cdot \mathbf{B}$, has been observed for all test problems we have solved.

From the comparison in Fig. 10, we conclude that it might be possible to preserve the divergence-free condition if we avoid applying the filter on the three components of the magnetic field equations. This requires that the filtering of the remaining components be enough to suppress spurious oscillations. The linear high order dissipation of the base scheme is of constant strength, and therefore does not affect the numerical divergence of 
the magnetic field. A proper choice of the strength of this high order dissipation helps keep spurious oscillations low when switching off the nonlinear filter on the magnetic field equations. (Here after refers to "without nonlinear filter on B".) In all of the numerical experiments (except the 1-D Riemann problem), linear filters are not activated on all of the field equations. We show results of this technique in Fig. 12, where the norm of the divergence of the magnetic field is plotted vs. time for the WAV66+AD8 method with (solid curves) and without (dashed curves) applying the nonlinear filter on the three magnetic field components of the MHD system. The dissipation was $d=0.005$ for the fully filtered computation, and $d=0.001$ for the computation without nonlinear filter on the magnetic field. Results from the three different uniform grids $101 \times 101,201 \times 201$, and $401 \times 401$ are shown for each method. The solutions at $401 \times 401$ grid points are shown for the methods with and without nonlinear filter on the magnetic field in Fig. 13.

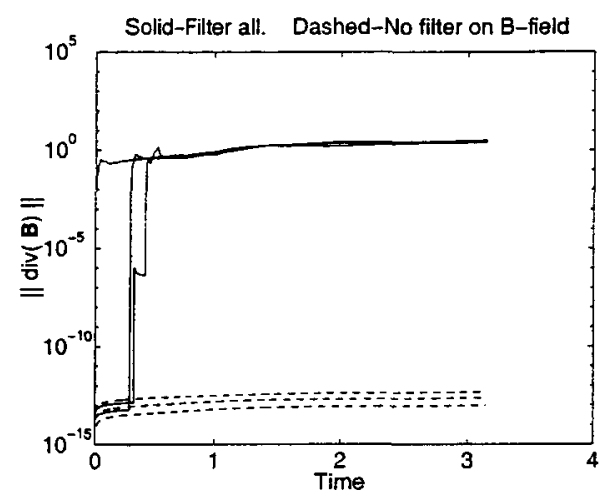

Fig. 12. Orszag-Tang problem. Divergence $\mathbf{B}$ versus time for uniform grids of $101 \times 101,201 \times 201$, and $401 \times 401$ with nonlinear filter on the $\mathbf{B}$ field equations (solid), without nonlinear filter on the $B$ field equations (dashed).

The solution without nonlinear filter on $\mathbf{B}$ has fewer oscillations than the filtered solution. This seems contradictory, but is due partially to the fact that the base scheme for the non-filtered $\mathbf{B}$ computation uses a different strength of an eighth-order linear numerical dissipation. More importantly, not filtering $\mathbf{B}$ preserves the $\nabla \cdot \mathbf{B}$ property (Fig. 12). In our companion paper Part II [21], we will illustrate that no filter on B might not always work for all problems. Sometimes we need to filter B to maintain nonlinear stability.

\subsection{A 2-D Riemann Problem}

The 2-D Riemann problem consists of four constant states at time zero, as shown in Fig. 14. The values of the initial states were taken from [5], and are given by

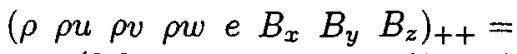

$$
\begin{aligned}
& \left(\begin{array}{llllllll}
0.9308 & 1.4557 & -0.4633 & 0.0575 & 5.0838 & 0.3501 & 0.9830 & 0.3050
\end{array}\right) \quad x>0 \quad y>0 \\
& \left(\rho \rho u \rho v \rho w e B_{x} \quad B_{y} B_{z}\right)_{-+}=
\end{aligned}
$$

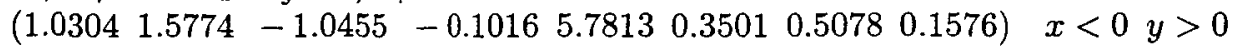

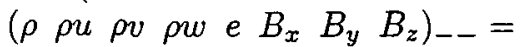

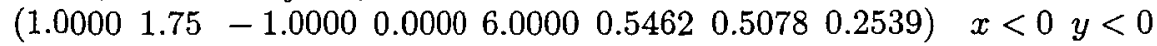

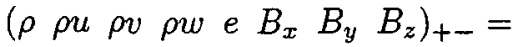

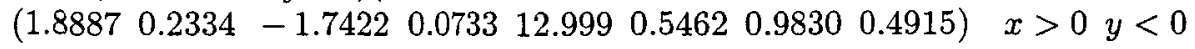



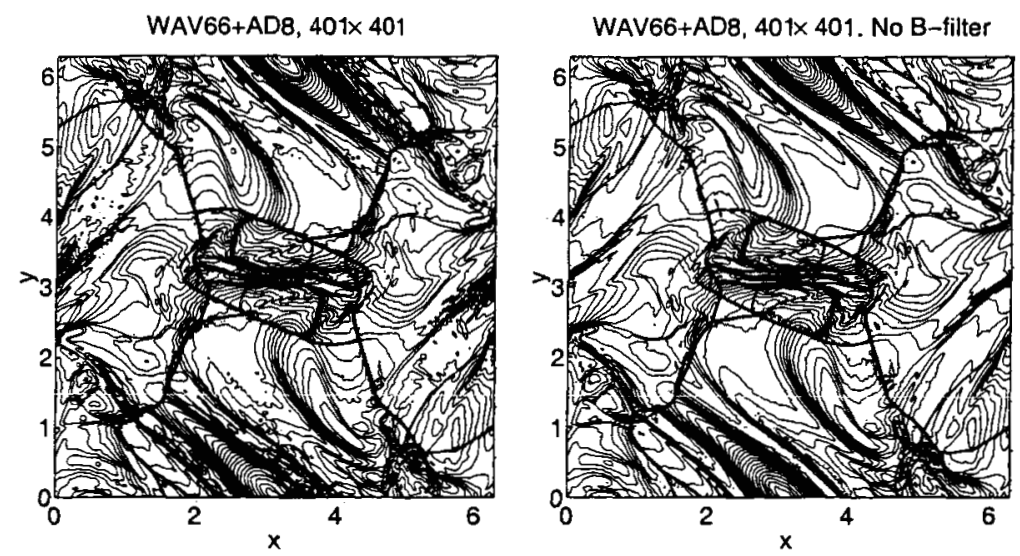

Fig. 13. Orszag-Tang problem. Density contours by WAV66+AD 8 with filter on the B field equations (left) and solution without filter on the $\mathbf{B}$ field equations (right) on magnetic field components.

The boundaries are treated as open boundaries. The problem is solved on the domain

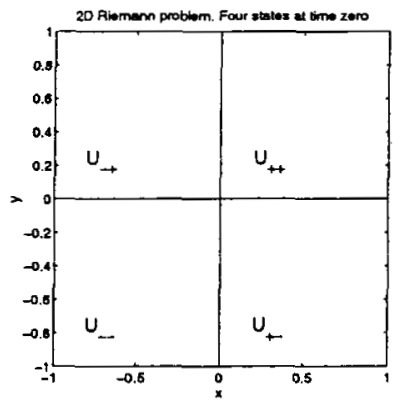

Fig. 14. Schematic of the initial data for the 2-D Riemann problem.

$-1<x<1,-1<y<1$ to time $\mathrm{T}=0.2$. The purpose of this example is to show the advantage of solving the conservative equations on high-resolution shock-capturing methods for a more than one-dimensional Riemann problem.

Density contours on grids of increasing refinement, computed by the second-order Harten-Yee TVD scheme applied to the conservative MHD equations are given in Fig. 15. The same computation solving the non-conservative MHD equations is shown in Fig. 16.

From the contours plots, there appear to have very little difference between the two methods. However, the $L^{2}$-norm of $\nabla \cdot \mathbf{B}$ vs. time shown in Fig. 17 illustrates that the solution of conservative equations has a smaller numerical $\nabla \cdot \mathbf{B}$ than the non-conservative equations. A contour plot of $\nabla \cdot \mathbf{B}$ is displayed in Fig. 18. The contours are placed at 

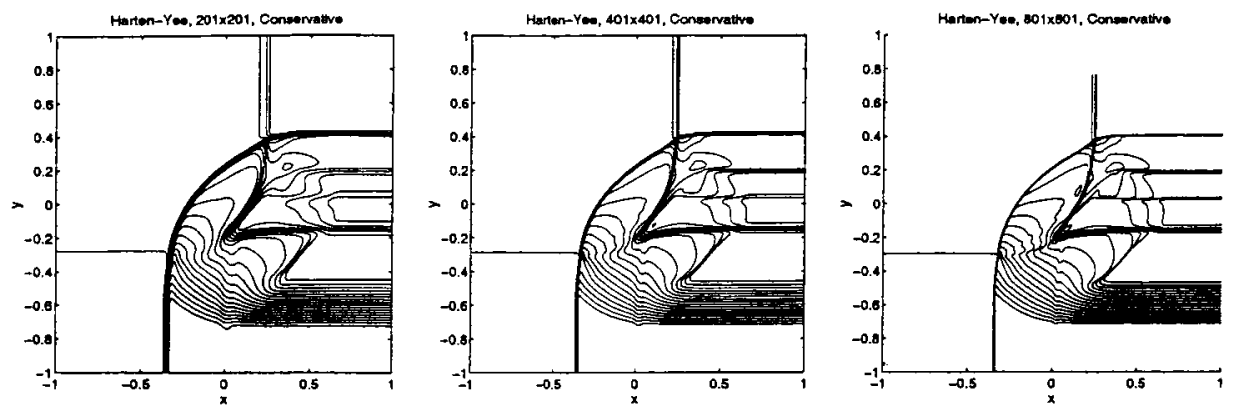

Fig. 15. Density contours of the 2-D Riemann problem. Grid refinement by Harten-Yee solving the conservative MHD equations.
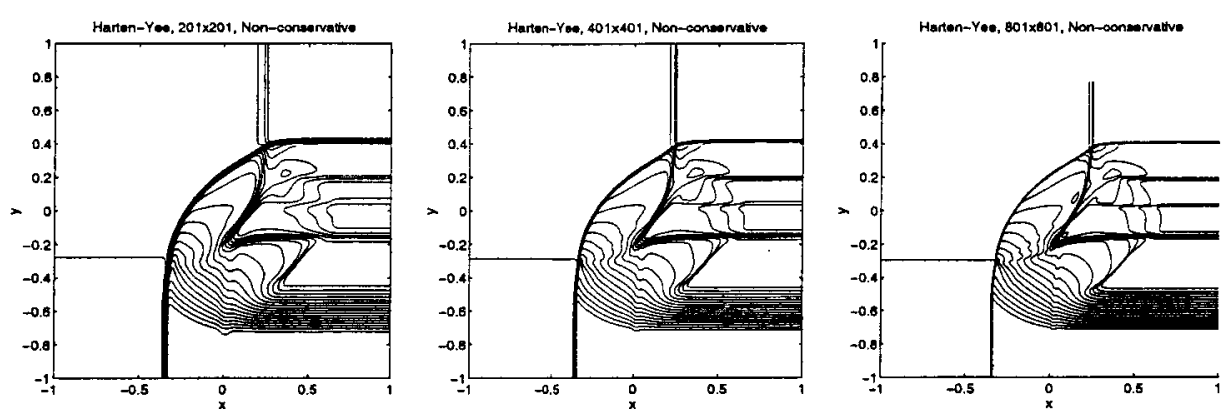

Fig. 16. Density contours of the 2-D Riemann problem. Grid refinement by Harten-Yee TVD solving the non-conservative MHD equations.

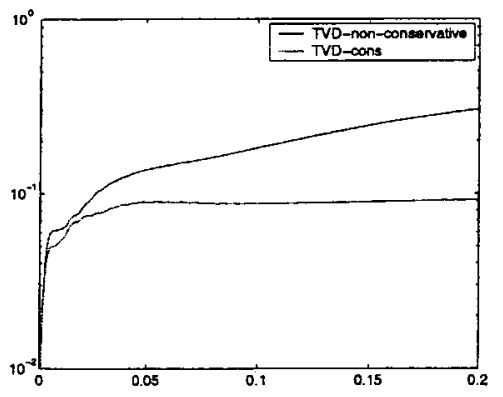

Fig. 17. 2-D Riemann problem. $L^{2}$ norm of $\nabla \cdot \mathbf{B}$ vs. time. Harten-Yee using a $201 \times 201$ grid solving both the conservative and non-conservative MHD equations. 
the same levels in the two plots. As expected, both methods have $\nabla \cdot \mathbf{B}$ numerical errors concentrated at the discontinuities. The better performance of the conservative method in this respect seems to be due to better handling of generation of $\nabla \cdot \mathbf{B}$ at discontinuities.

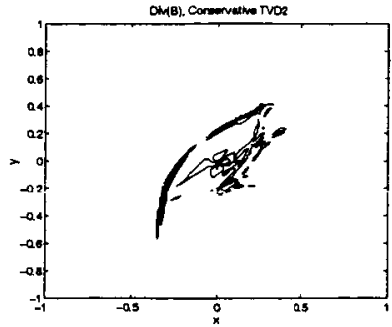

(a) Conservative

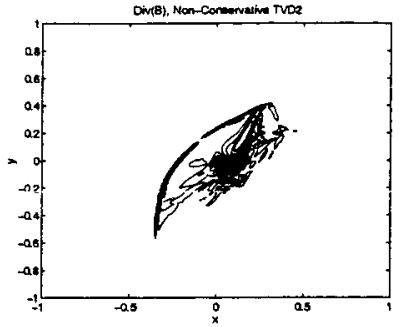

(b) Non-conservative

Fig. 18. 2-D Riemann problem. $\nabla \cdot \mathbf{B}$ contours by Harten-Yee solving both the conservative and non-conservative MHD for a $201 \times 201$ uniform grid.

\subsection{Vortex Pairing}

Vortex pairing in a mixing layer was simulated in [18], where the compressible NavierStokes equations were solved. The set-up of the problem is given in Fig. 19. The Navier-

\section{Vortex Pairing in a Time-Developing Mixing Layer \\ $\left(M_{c}=0.8, R e=1000, T_{R}=300 \mathrm{~K}\right.$, Prandt $\left.\#=0.72\right)$}

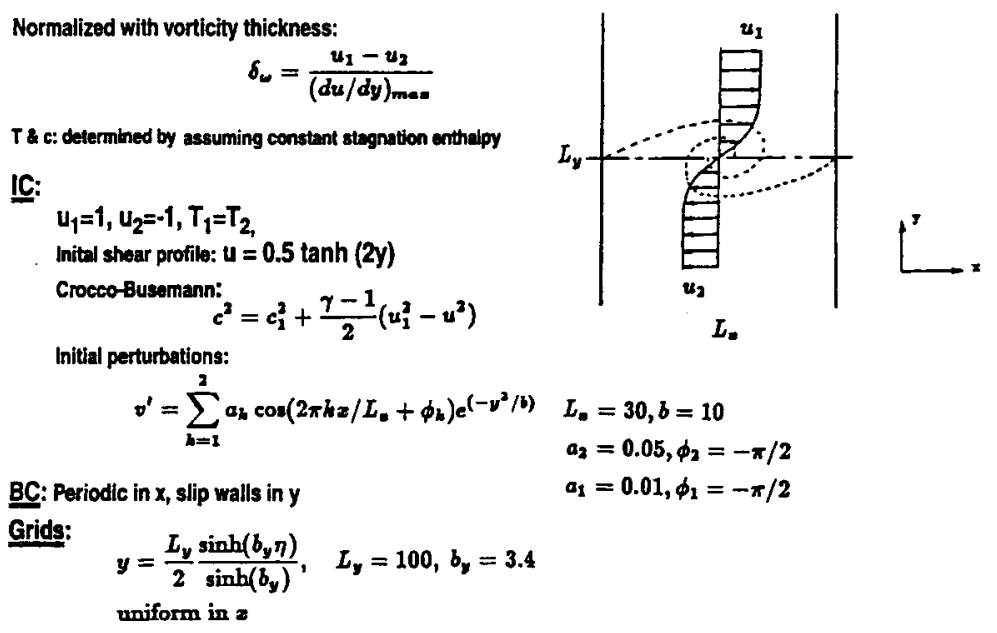

Fig. 19. Schematic of the vortex pairing problem.

Stokes equations are solved with a Reynolds number of 1000 . The grid is mildly stretched 
towards the line $y=0$ with a sine-hyp stretching function. The boundary conditions are periodic in the $x$-direction and open on upper and lower $y$-boundaries.

As seen in Fig. 19, the initial flow consists of two constant states separated by a mixing layer. A small perturbation, which generates two vortices, is introduced at time zero. The two vortices are approaching each other. The problem is solved to a time when the vortices have merged.

Here, generalization of the hydrodynamic problem to MHD by imposing an initially constant magnetic field in the $x$-direction, $B_{x}=0.1$, is used as a test case. In addition, we are solving the non-ideal MHD equations in the sense that a resistivity coefficient of $\sigma=100$ and the same Reynolds number (1000) and Prandtl number (0.72) that were used in the non-magnetic case [18] are used here. The non-ideal MHD terms are not shown in (1). See [1] for the form. The purpose of this numerical experiment is to show the performance of the scheme for an non-ideal MHD.

The viscous terms are discretized by the sixth-order central differencing for WAV66, ACM66 and WENO5, and second-order central differencing for MUSCL and Harten and Yee.

We solve the problem to time 90 . Figure 20 shows temperature contours at time 90 of the solution, computed by the Harten-Yee second-order TVD scheme. The grid is refined from $201 \times 201$ grid points to $1601 \times 1601$ points. Grid convergence is reached without problems, due to a fairly small Reynolds number and conductivity.

The advantage of a high order method to resolve the scales is seen in Fig. 21, where a grid convergence study for the sixth-order WAV66+AD8 method is shown. The dissipations $d=0.001$ and the CFL number 0.6 were used. By comparing Figs. 20 and 21, we conclude that the sixth-order scheme captures all features of the flow correctly with $201 \times 201$ grid points. The solutions (figures not shown) using ACM66 (with or without AD8) is similar to WAV66+AD8 except ACM66 is more stable than WAV66+AD8. WAV66 (without AD8) is not stable. The cause of the difference is very complex and is currently under investigation since the non-ideal MHD is an incompletely parabolic PDE system. The findings will be reported in a future publication. The second-order scheme needs to use $401 \times 401$ points to obtain a similar quality of the solution. This is in agreement with similar investigations for non-MHD computations [13]. Finally, in Fig. 22, we show a comparison of the development of $L^{2}$ norm of $\nabla \cdot \mathbf{B}$ in time for the second-order accurate Harten-Yee scheme, and the sixth-order WAV66+AD8 scheme. Computations using the WENO5 schemes for this problem require an excessively small time step and are extremely slow to converge (solution not shown).

\section{Concluding Remarks}

The generalization of our low dissipative high order schemes for hydrodynamics equations to MHD equations has been presented. Three of the four features of the present MHD schemes summarized in the abstract are validated by typical 1-D and 2-D MHD test cases. The new method of defining high-resolution shock-capturing schemes for the conservative MHD equations exhibits smaller $\nabla \cdot \mathrm{B}$ numerical error when compared to the standard numerical method for solving the non-conservative MHD equations. The approach of using the non-conservative eigensystem when solving the conservative equations is also applicable 

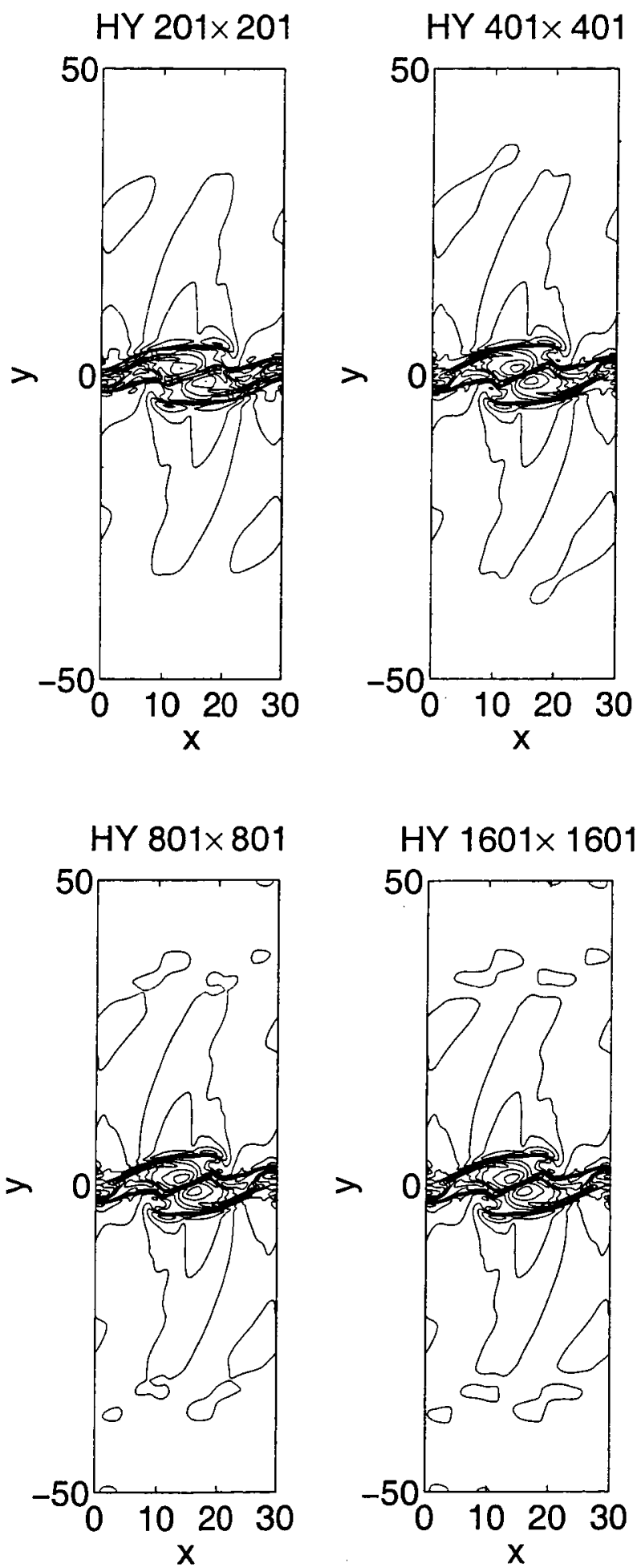

Fig. 20. Temperature contours for the vortex pairing problem. Grid refinement by Harten-Yee solving the conservative MHD equations. 


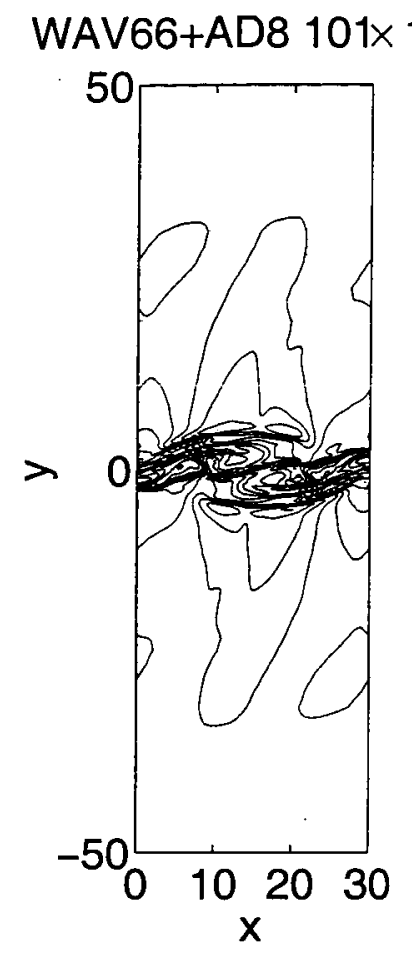

WAV66+AD8 201× 201

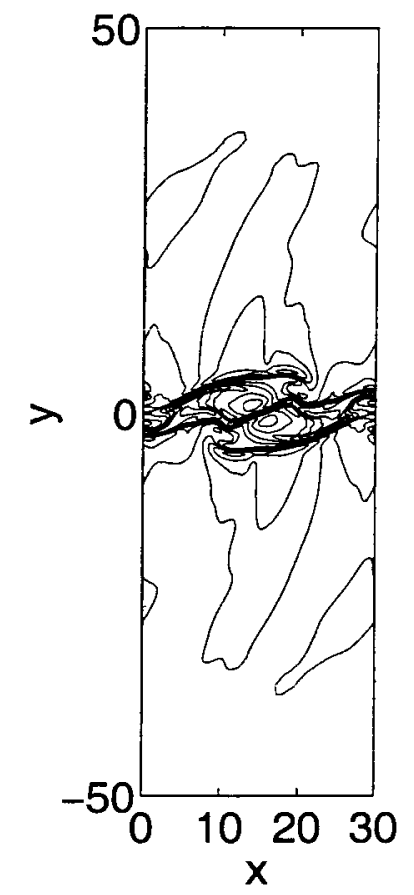

WAV66+AD8 401× 401
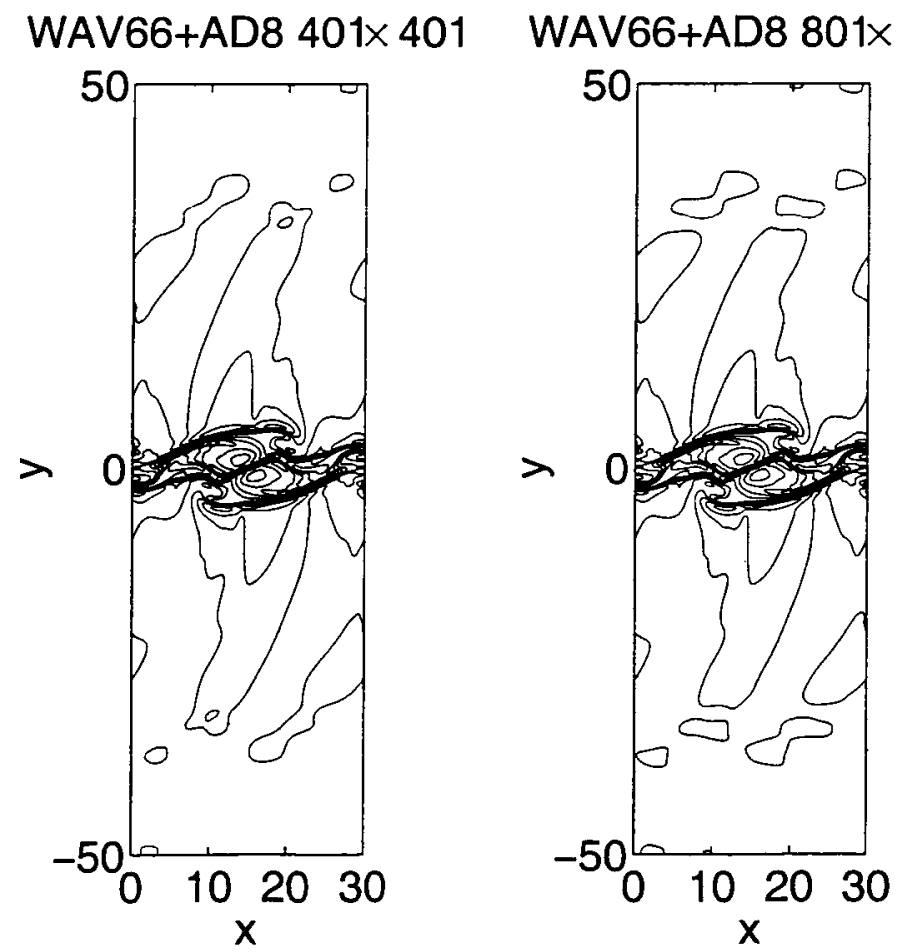

Fig. 21. Temperature contours of the vortex pairing problem. Grid refinement for the WAV66+AD8 scheme. Conservative MHD equations. 


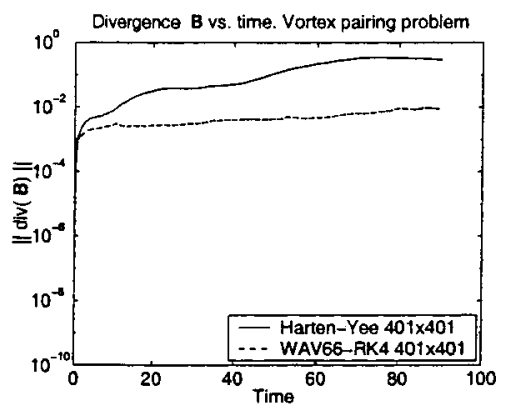

Fig. 22. Vortex pairing problem. $\nabla \cdot \mathrm{B}$ vs. time. Harten-Yee (solid) WAV66+AD8 (dashed).

in the context of commonly used shock-capturing schemes for the MHD equations in the literature.

More importantly, the filter approach of our new method provides a natural and efficient way to minimize the $\nabla \cdot \mathbf{B}$ numerical error. It also offers a variety of options to cater to different flow types of the problem. Since there are many variants in the minimization of the divergence of the magnetic field $(\nabla \cdot \mathbf{B})$ numerical error, Part II of the companion paper [21] is devoted to extensive comparison of these variants. In yet another forthcoming paper, Part III, complex multiscale high speed turbulent astrophysical applications will be sought.

\section{Acknowledgment}

Part of this work was performed while the first author was a RIACS visiting scientist, NASA Ames Research Center.

\section{References}

1. Reference for the non-ideal MHD ????

2. M. Brio and C.C. Wu, An Upwind Differencing Scheme for the Equations of Ideal Magnetohydrodynamics, J. Comput. Phys., 75 (1988), pp. 400-422.

3. P. Cargo and G. Gallice, Roe Matrices for Ideal MHD and Systematic Construction of Roe Matrices for Systems of Conservation Laws, J. Comput. Phys., 136 (1997), pp. 446-466.

4. W. Dai and P. R. Woodward, A Simple Finite Difference Scheme for Multidimensional Magnetohydrodynamical Equations J. Comput. Phys., 142 (1998), pp. 331-369.

5. A. Dedner, F. Kemm, D. Kröner, C.-D. Munz, T. Schnitzer, and M. Wesenberg, Hyperbolic Divergence Cleaning for the MHD Equations, J. Comput. Phys., 175 (2002), pp. 645-673.

6. H. De Sterck, Multi-Dimensional Upwind Constrained Transport on Unstructured Grids for Shallow Water Magnetohydrodynamics, AIAA Paper 2001-2623, (2001).

7. C.R. Evans and J.F. Hawley, Simulation of Magnetohydrodynamic Flows: A Constrained Transport Method, Astrophys. J. 332, (1988), pp. 332-???.

8. G. Gallice, Systéme dÉuler-Poisson, Magnétohydrodynamique et Schemeas de Roe, PhD Thesis, L'Université Bordeaux I, 1997.

9. G.-S. Jiang and C.-W. Shu, Efficient Implementation of Weighted ENO schemes, J. Comput. Phys., 126 (1996), pp. 202-228. 
10. K.G. Powell, P.L. Roe, T.J. Linde, T.I. Gombosi and D.L. De Zeeuw, A Solution-Adaptive Upwind Scheme for Ideal Magnetohydrodynamics, J. Comput. Phys., 154 (1999), pp. 284-309.

11. W. Dai and P.R. Woodward, A simple Finite Difference Scheme for Multidimensional Magnetohydrodynamical Equations, J. Comput. Phys., 142, (1998), pp. 331-369.

12. B. Sjögreen and H. C. Yee, Multiresolution Wavelet Based Adaptive Numerical Dissipation Control for Shock-Turbulence Computation, RIACS Technical Report TR01.01, NASA Ames research center (Oct 2000), to appear, J. Scient. Computing

13. B. Sjögreen and H. C. Yee, Grid Convergence of High Order Methods for Multiscale Complex Unsteady Viscous Compressible Flows, RIACS Technical Report TR01.06, April, 2001, NASA Ames research center; AIAA 2001-2599, Proceedings of the 15th AIAA CFD Conference, June 11-14, 2001, Anaheim, CA., also, J. Comput. Phys., 185, (2003), pp. 1-26.

14. B. Sjögreen and H. C. Yee, Low Dissipative High Order Numerical Simulations of Supersonic Reactive Flows, RIACS Technical Report TR01-017, NASA Ames Research Center (May 2001); Proceedings of the ECCOMAS Computational Fluid Dynamics Conference 2001, Swansea, Wales, UK, September 4-7, 2001.

15. B. Sjögreen and H. C. Yee, Analysis of High Order Difference Methods for Multiscale Complex Compressible Flows, Proceedings of the 9th International Conference on Hyperbolic Problems, March 25-29, 2002, Pasadena, CA.

16. G. Tóth, The div $B=0$ Constraint in Shock-Capturing Magnetohydrodynamics Codes, J. Comput. Phys., 161, (2000), pp. 605-652.

17. H.C. Yee, A Class of High-Resolution Explicit and Implicit Shock-Capturing Methods, VKI Lecture Series 1989-04, March 6-10, 1989, also NASA TM-101088, Feb. 1989.

18. H.C. Yee, N.D. Sandham, N.D., and M.J. Djomehri, Low Dissipative High Order ShockCapturing Methods Using Characteristic-Based Filters, J. Comput. Phys., 150 (1999) pp. 199-238.

19. H.C. Yee, M. Vinokur, M., and M.J. Djomehri, Entropy Splitting and Numerical Dissipation, J. Comput. Phys., 162 (2000) pp. 33-81 (2000).

20. H.C. Yee and B. Sjögreen, Designing Adaptive Low Dissipative High Order Schemes for LongTime Integrations, Turbulent Flow Computation, (Eds. D. Drikakis \& B. Geurts), Kluwer Academic Publisher (2002); also RIACS Technical Report TR01-28, Dec. 2001.

21. H.C. Yee and B. Sjögreen, Efficient Low Dissipative High Order Schemes for Multiscale MHD Flows, II: Minimization of $\nabla \cdot \mathbf{B}$ Numerical Error, RIACS Technical Report, NASA Ames Research Center, 2003. A condensed version appears in the Proceedings of the International Conference on High Performance Scientific Computing, March 10-14, 2003, Hanoi, Vietnam.

22. K.S.Yee, Numerical solution of initial boundary value problems involving Maxwell's equations in isotropic media, IEEE Trans. Antennas Propagat., 14 (1966), pp. 302-307. 